\title{
USCAP Long Course 2002: New Concepts in Gastrointestinal Pathology
}

Since the last USCAP Long Course on Gastrointestinal Pathology in 1988, there has been continued explosive growth in gut endoscopic procedures, with a concomitant increase in biopsy specimens from the esophagus, stomach, small intestine, and colon. In fact, gastrointestinal specimens now account for up to $25 \%$ of all surgical pathology material in many clinical practices. The primary objective of this course has been the presentation of updated reviews of common as well as new, controversial, and diagnostically challenging inflammatory and neoplastic conditions of the gastrointestinal tract to the community of diagnostic surgical pathologists. In addition to routine histopathology, the use of immunohistochemical, molecular biological, and cytogenic studies for diagnosis and prognosis of particular diseases has been critically evaluated, and the results incorporated into the appropriate individual presentations. As a result, the diagnosis and outcome of the conditions under discussion. I hope we have been successful in achieving this goal.

Donald A. Antonioli, MD 